\title{
Comment on "Clinical Dementia Rating Orientation Score as an Excellent Predictor of the Progression to Alzheimer's Disease in Mild Cognitive Impairment"
}

\author{
Tomoyuki Kawada $\bowtie$ \\ Department of Hygiene and Public Health, Nippon Medical School, Tokyo, Japan
}

Sir,

Kim et al. ${ }^{1}$ examined the usefulness of the Clinical Dementia Rating (CDR) for predicting Alzheimer's disease (AD) dementia progression in 59 elderly subjects with mild cognitive impairment (MCI). By logistic regression analysis, odds ratio [95\% confidence interval (CI)] of CDR Orientation subscale for $\mathrm{AD}$ dementia progression was 12.3 (3.5-43.8). The authors recommended CDR Orientation subscale score for predicting $\mathrm{AD}$ dementia progression in elderly subjects with MCI. I have some concerns about their study.

First, Xue et al. ${ }^{2}$ investigated risk factors for progression to $\mathrm{AD}$ and death in 437 elderly subjects with MCI. They adopted Cox model and adjusted hazard ratios (95\% CIs) of female, older age, reading occasionally, current smoking, light-moderate alcohol drinking, cerebrovascular disease, hyperlipidemia and diabetes for transition from MCI to AD were $1.82(1.20-$ 2.77), 3.09 (1.81-5.25), 1.79 (1.11-2.89), 1.74 (1.15-2.65), 2.24 (1.42-3.53), 2.70 (1.68-4.34), 1.87 (1.16-3.02), and 1.81 (1.18$2.77)$, respectively. These significant factors should be considered as contributors of $\mathrm{AD}$ dementia progression in elderly subjects with MCI.
Second, Kim et al. ${ }^{1}$ handled a limited number of patients with MCI. From 59 subjects, 22 subjects progressed from $\mathrm{MCI}$ to $\mathrm{AD}$ after the 2 years of follow-up. I suppose that the wide range of $95 \% \mathrm{CI}$ in odds ratio would reflect unstable estimation and summing-up of samples are recommend. ${ }^{3}$

Finally, a simple and easy clinical measure is important for predicting $\mathrm{AD}$ dementia progression in elderly subjects with MCI. But statistical significance by logistic regression analysis and discriminant ability differs, and combination of several predictors should be considered for the progression.

\section{REFERENCES}

1. Kim JW, Byun MS, Sohn BK, Yi D, Seo EH, Choe YM, et al. Clinical dementia rating orientation score as an excellent predictor of the progression to alzheimer's disease in mild cognitive impairment. Psychiatry Investig 2017;14:420-426.

2. Xue H, Sun Q, Liu L, Zhou L, Liang R, He R, et al. Risk factors of transition from mild cognitive impairment to Alzheimer's disease and death: a cohort study. Compr Psychiatry 2017;78:91-97.

3. Peduzzi P, Concato J, Kemper E, Holford TR, Feinstein AR. A simulation study of the number of events per variable in logistic regression analysis. J Clin Epidemiol 1996;49:1373-1379.
Received: October 13, 2017 Accepted: November 6, 2017 Available online: November 29, 2017

$\triangle$ Correspondence: Tomoyuki Kawada, MD

Department of Hygiene and Public Health, Nippon Medical School, 1-1-5 Sendagi, Bunkyo-Ku, Tokyo 113-8602, Japan

Tel: +81-3-3822-2131, Fax: +81-3-5685-3065, E-mail: kawada@nms.ac.jp

(c) This is an Open Access article distributed under the terms of the Creative Commons Attribution Non-Commercial License (http://creativecommons.org/licenses/bync/4.0) which permits unrestricted non-commercial use, distribution, and reproduction in any medium, provided the original work is properly cited. 